\title{
THE GAUSS CONSTRAINT IN THE EXTENDED LOOP REPRESENTATION
}

\author{
Cayetano Di Bartolo \\ Departamento de Física, Universidad Simón Bolívar, Apartado 89000, \\ Caracas 1080-A, Venezuela.
}

\begin{abstract}
The Gauss constraint in the extended loop representation for quantum gravity is studied. It is shown that there exists a sector of the state space that is rigorously gauge invariant without the generic convergence issues of the extended holonomies.
\end{abstract}

pacs: $11.15 .-\mathrm{q}, 04.60 .-\mathrm{m}$

The Ashtekar variables [1, 2] present general relativity as a gauge theory. As a consequence, it is possible to quantize the theory in the loop space [3]. In this representation, the wave functions are loop dependent. All the information relevant to the loop is found in its 'multitangents fields' which can be thought of as loop coordinates «4. The loop representation poses some advantages over the connection representation: the Gauss constraint is solved in the former, and furthermore, using knot invariant wave functions, the diffeomorphism constraint is solved.

Recently, the gravity extended loop representation was introduced [5, 6] as a generalization of the loop representation. In this new representation, the wave function domain is the extended loop space. This vector space of infinite dimension includes the loop coordinates as a particular subset. The extended loop representation has several interesting features: it allows to deal with regularization problems of wave functions which are inherent to the loop 
representation; it supplies [7] a new context in which to deal with constraint regularization and renormalization, and it supplies new calculation tools to handle loop dependent objects.

In this work we will discuss the problem of the gauge covariance of the extended representation, some aspects of this problem were pointed out and discussed in references [7], 8]. We shall show here that there exists a sector of the state space which is gauge invariant. This sector contains most of the wave functions know in the extended loop representation.

In order to deal with the extended loop representation, it is useful to define a number of vector spaces. We define the vector space $\mathcal{E}$ of infinite dimension as the space of all the linear combinations of a linearly independent base of vectors $\boldsymbol{\delta}_{\dot{\nu}}$ (the subindex $\dot{\nu}$ labels the elements of the base),

$$
\boldsymbol{E} \in \mathcal{E} \leftrightarrow \boldsymbol{E}=\boldsymbol{\delta}_{\dot{\nu}} E^{\dot{\nu}}
$$

The dotted Greek indexes correspond to an ordered set of Greek indexes $\dot{\nu}=\nu_{1} \nu_{2} \cdots \nu_{n}$ where $n=n(\dot{\nu})$ is the number of elements in the set. A Greek index has a discrete part and a continuous part, $\mu=(a, x)$ with $a \in\{1,2,3\}$ and $x \in R^{3}$. We are adopting the generalized Einstein convention for repeated indexes

$$
\varphi_{\mu} E^{\mu}=\sum_{i=1}^{3} \int d^{3} x \varphi_{i x} E^{i x} \quad \text { and } \quad \varphi_{\dot{\mu}} E^{\dot{\mu}}=\varphi E+\sum_{n=1} \varphi_{\mu_{1} \cdots \mu_{n}} E^{\mu_{1} \cdots \mu_{n}} .
$$

In (11), $E^{\nu_{1} \cdots \nu_{n}}$ is the component of range $\mathrm{n}$ of $\boldsymbol{E}$. The components of the vector $\boldsymbol{\delta}_{\dot{\nu}}$ are:

$$
\delta_{\dot{\nu}}^{\dot{\mu}}= \begin{cases}0 & \text { if } n(\dot{\mu}) \neq n(\dot{\nu}) \\ 1 & \text { if } n(\dot{\mu})=n(\dot{\nu})=0 \\ \delta_{\nu_{1}}^{\mu_{1}} \ldots \delta_{\nu_{n}}^{\mu_{n}} & \text { if } n=n(\dot{\mu})=n(\dot{\nu}) \geq 1\end{cases}
$$

where

$$
\delta_{b y}^{a x} \equiv \delta_{b}^{a} \delta(x-y)
$$

In the space $\mathcal{E}$, we introduce the vector product:

$$
\boldsymbol{E}_{1} \times \boldsymbol{E}_{2} \equiv \boldsymbol{\delta}_{\dot{\mu} \dot{\nu}} E_{1}^{\dot{\mu}} E_{2}^{\dot{\nu}}
$$


The extended loop space $\llbracket 4 \mathcal{E}_{y}$ is the vector subspace of elements of $\mathcal{E}$ that satisfy the following differential constraint:

$$
\frac{\partial}{\partial x^{a}} E^{\dot{\alpha} a x \dot{\beta}}=\delta_{\dot{\nu} a x}^{\dot{\alpha}} E^{\dot{\nu} a x \dot{\beta}}-\delta_{a x \dot{\nu}}^{\dot{\beta}} E^{\dot{\alpha} a x \dot{\nu}}+\delta_{x, y}\left[\delta^{\dot{\alpha}} E^{\dot{\beta}}-\delta^{\dot{\beta}} E^{\dot{\alpha}}\right]
$$

where there is a sum over "a" and " $y$ " is the origin of the extended loop. This equation can be written as follows (without explicitly indicating the $\delta_{\dot{\nu}}^{\dot{\mu}}$ quantities):

$$
\frac{\partial}{\partial x^{a}} E^{a_{1} x_{1} \cdots a_{i} x_{i} a x a_{i+1} x_{i+1} \cdots a_{n} x_{n}}=\left[\delta\left(x_{i}-x\right)-\delta\left(x_{i+1}-x\right)\right] E^{a_{1} x_{1} \cdots a_{n} x_{n}}
$$

where $0 \leq i \leq n$ and $x_{0} \equiv x_{n+1} \equiv y$. The differential constraint relates the components of range $n+1$ and range $n$ of $\mathbf{E}$, and basically, indicates that the completely transverse parts of $\mathbf{E}$ are free, i.e., to solve the constraint means to establish an isomorphism between $\mathcal{E}_{y}$ and the transverse vector space [4]. It can be shown that the product(5) is closed in $\mathcal{E}_{y}$. The vectors in $\mathcal{E}_{y}$ with the positive null range component together with the product (5) define the extended loop group.

We define $\mathcal{N}$ as the space constituted by the linear applications $\varphi, \varphi: \mathcal{E} \rightarrow \mathcal{Q}$, that satisfy

$$
\exists \mathrm{m} \text { integer } / \varphi(\boldsymbol{E})=\varphi_{\dot{\mu}} E^{\dot{\mu}}=\sum_{n=0}^{m} \varphi_{\mu_{1} \cdots \mu_{n}} E^{\mu_{1} \cdots \mu_{n}} .
$$

The extended loop representation for a gauge theory or for quantum gravity is dual to the connection representation, and it is formally obtained from the extended loop transform,

$$
\varphi(\boldsymbol{E})=\int D A \hat{\varphi}(A) W_{\boldsymbol{E}}[A]
$$

where

$$
W_{\boldsymbol{E}}[A]=\operatorname{Tr}\left[A_{\dot{\mu}}\right] E^{\dot{\mu}}
$$

is the Wilson extended functional and $A_{\dot{\mu}}$ denotes the product

$$
A_{a_{1} x_{1} \cdots a_{n} x_{n}}=A_{a_{1}}\left(x_{1}\right) \cdots A_{a_{n}}\left(x_{n}\right) .
$$

The $\boldsymbol{E}$ coordinate in $(10)$ is a vector in $\mathcal{E}_{y}$. However, the origin of extended coordinate is not relevant. Due to the trace in (10), the cyclic part of $\boldsymbol{E}$ is the only one that contributes, this 
part loses every notion of the origin; in effect, it inherits from (6) the following differential constraint without origin:

$$
V_{x \dot{\mu}}^{\dot{\alpha}} E^{\dot{\mu}}=-\frac{\partial}{\partial x^{a}} E^{(a x \dot{\alpha})_{c}}+\left[\boldsymbol{\delta}_{\dot{\nu}}, \boldsymbol{\delta}_{a x}\right]^{\dot{\alpha}} E^{(a x \dot{\nu})_{c}}=0
$$

with

$$
V_{x \dot{\mu}}^{\dot{\alpha}} \equiv-\frac{\partial}{\partial x^{a}} \boldsymbol{\delta}_{\dot{\mu}}^{(a x \dot{\alpha})_{c}}+\left[\boldsymbol{\delta}_{\dot{\nu}}, \boldsymbol{\delta}_{a x}\right]^{\dot{\alpha}} \boldsymbol{\delta}_{\dot{\mu}}^{(a x \dot{\nu})_{c}}
$$

where there is a sum in 'a' and no integration in ' $x$ ', the notation ()$_{c}$ meaning the sum of all the cyclic permutations of indices and the commutator is formed with the product ' $X$ '. Formally, the differential constraint guarantees the gauge invariance of $W_{\boldsymbol{E}}[A]$ and of the theory. However, the functional (10) is not well defined for all the elements of the extended loop space. There exist pairs of connections and vectors of the extended loop space for which the infinite sum in (10) does not converge; but we shall see that there is a sector in the state space where this problem is not important.

Under the infinitesimal gauge transformation,

$$
\left(\delta A_{a x}\right)=\Lambda_{x, a}+A_{a x} \Lambda_{x}-\Lambda_{x} A_{a x}
$$

the quantity $A_{\dot{\mu}}$ transforms according to

$$
\delta A_{\mu_{1} \cdots \mu_{n}}=\sum_{h=1}^{n} A_{\mu_{1} \cdots \mu_{h-1}}\left(\delta A_{\mu_{h}}\right) A_{\mu_{h+1} \cdots \mu_{n}}=\delta_{\mu_{1} \cdots \mu_{n}}^{\dot{\alpha} a x \dot{\beta}} A_{\dot{\alpha}}\left(\delta A_{a x}\right) A_{\dot{\beta}} .
$$

Its trace satisfies

$$
\delta \operatorname{Tr}\left[A_{\dot{\mu}}\right]=\delta_{\dot{\mu}}^{\dot{\alpha} a x \dot{\beta}} \operatorname{Tr}\left[A_{\dot{\beta} \dot{\alpha}}\left(\delta A_{a x}\right)\right]=\delta_{\dot{\mu}}^{\dot{\alpha} a x \dot{\beta}} \delta_{\dot{\beta} \dot{\alpha}}^{\dot{\nu}} \operatorname{Tr}\left[A_{\dot{\nu}}\left(\delta A_{a x}\right)\right]
$$

Using in this expression, the identity

$$
\delta_{\dot{\mu}}^{\dot{\alpha} a x \beta} \delta_{\dot{\beta} \dot{\alpha}}^{\dot{\nu}}=\delta_{\dot{\mu}}^{(a x \dot{\nu})_{c}}
$$

and replacing $\delta A_{a x}$ from (14), we get

$$
\delta \operatorname{Tr}\left[A_{\dot{\mu}}\right]=\operatorname{Tr}\left[-\Lambda_{x} A_{\dot{\nu}} \frac{\partial}{\partial x^{a}}+\Lambda_{x} A_{\dot{\alpha}}\left(\delta_{\dot{\nu} a x}^{\dot{\alpha}}-\delta_{a x \dot{\nu}}^{\dot{\alpha}}\right)\right] \delta_{\dot{\mu}}^{(a x \dot{\nu})_{c}}=\operatorname{Tr}\left[\Lambda_{x} A_{\dot{\alpha}}\right] V_{x \dot{\mu}}^{\dot{\alpha}}
$$


We introduce the gauge dependent quantities

$$
\varphi_{\mu_{1} \cdots \mu_{n}}^{I_{1} \cdots I_{n}} \equiv \int D A \hat{\varphi}(A) A_{\mu_{1}}^{I_{1}} \cdots A_{\mu_{n}}^{I_{n}}
$$

and

$$
\varphi_{\mu_{1} \cdots \mu_{n}} \equiv \varphi_{\mu_{1} \cdots \mu_{n}}^{I_{1} \cdots I_{n}} \operatorname{Tr}\left(T^{I_{1}} \cdots T^{I_{n}}\right)=\int D A \hat{\varphi}(A) \operatorname{Tr}\left(A_{\mu_{1}} \cdots A_{\mu_{n}}\right)
$$

where the $T^{I}$ are the gauge group generators. Because of (18), $\varphi_{\dot{\mu}}$ transforms according to

$$
\delta \varphi_{\dot{\mu}}=\int D A \operatorname{Tr}\left[\Lambda_{x} A_{\dot{\alpha}}\right] V_{x \dot{\mu}}^{\dot{\alpha}}
$$

under the gauge change (14). We shall suppose now that $\hat{\varphi}(A)$ is such that in some gauge $\mathrm{G}$ the following "cut condition" is satisfied:

$$
\exists M / \varphi_{\mu_{1} \cdots \mu_{n}}^{I_{1} \cdots I_{n}}=0 \quad \forall n>M
$$

This implies that in the gauge $\mathrm{G}$ the function

$$
\varphi(\boldsymbol{E})=\int D A \hat{\varphi}(A) \operatorname{Tr}\left[A_{\dot{\mu}}\right] E^{\dot{\mu}}=\varphi_{\dot{\mu}} E^{\dot{\mu}},
$$

is not dependent on the components of range $n(\dot{\mu})>M$ in $\boldsymbol{E}$. Next, let us show that $\varphi(\boldsymbol{E})$ is invariant under infinitesimal gauge changes. We shall only take gauge transformations for which $\Lambda_{x}$ can be expanded in a 'power series' in the connection,

$$
\Lambda_{x}=\lambda_{x}+\lambda_{x}^{J, \mu} A_{\mu}^{J}+\cdots
$$

From (21) we have

$$
\delta \varphi(\boldsymbol{E})=\int D A T r\left[\Lambda_{x} A_{\dot{\alpha}}\right] V_{x \dot{\mu}}^{\dot{\alpha}} E^{\dot{\mu}}
$$

In this expression, as a consequence of the "cut condition" the sum in $\dot{\alpha}$ is finite, $(\operatorname{Max} n(\dot{\alpha})=M)$, and from the definition of $V_{x \dot{\mu}}^{\dot{\alpha}}$, we have that $\operatorname{Max} n(\dot{\mu})=M+1$, i.e., the sum in (25) involves a finite number of ranges of $\boldsymbol{E}$, and because (12), we have

$$
\delta \varphi(\boldsymbol{E})=0
$$


The functionals $\varphi(\boldsymbol{E})=\varphi_{\dot{\mu}} E^{\dot{\mu}}$, which satisfy the "cut condition", are gauge invariant, and belong to $\mathcal{N}$. These functionals satisfy Mandelstam identities which reflect the specific structure of the gauge group [6]. In effect, for SU(2), the Mandelstam identities -which relates group generator product traces- lead to the following relations:

$$
\begin{aligned}
\varphi_{\dot{\mu} \dot{\nu}}= & \varphi_{\dot{\nu} \dot{\mu}} \\
\varphi_{\dot{\mu}} & =\varphi_{\dot{\mu}} \\
\varphi_{\dot{\mu} \dot{\nu} \dot{\gamma}}+\varphi_{\dot{\mu} \dot{\mu} \dot{\gamma}} & =\varphi_{\dot{\nu} \dot{\mu} \dot{\gamma}}+\varphi_{\dot{\nu} \dot{\mu} \bar{\gamma}}
\end{aligned}
$$

where to overline a set of Greek indexes means to invert the order and the multiplication by a sign, as follows:

$$
\overline{\delta_{\dot{\nu}}^{\overline{\mu_{1} \cdots \mu_{n}}}} \equiv \delta_{\dot{\nu}}^{\mu_{1} \cdots \mu_{n}} \equiv(-1)^{n} \delta_{\dot{\nu}}^{\mu_{n} \cdots \mu_{1}}
$$

The set of functionals that belong to $\mathcal{N}$ and that satisfy the identities (27) contains a sector, gauge invariant, of the state space of the quantum gravity extended loop representation.

In quantum gravity, the wave functions, $\varphi(\boldsymbol{E})=\varphi_{\dot{\mu}} E^{\dot{\mu}}$, must be invariant under diffeomorphism. This implies that by evaluating $\varphi(\boldsymbol{E})$ in the coordinate of a loop $\Gamma, \boldsymbol{E}=\boldsymbol{X}(\Gamma)$, a knot invariant is obtained. Reciprocally, if we have a knot invariant of the form

$$
\Psi(\Gamma)=\Psi^{\prime}(\boldsymbol{X}(\Gamma))=\sum_{n=0}^{M} \Psi_{\mu_{1} \cdots \mu_{n}} \boldsymbol{X}(\Gamma)^{\mu_{1} \cdots \mu_{n}},
$$

the function $\Psi^{\prime}(\boldsymbol{E})$ belongs to the state space in the extended loop representation. If the perturbative expansion of the expectation value of the Wilson functional in the ChernSimons theory is considered, every one of orders of the expansion has the form [9] (29). An example of a knot invariant which is not a finite linear combination of the form (29) is the exponential of the Gauss invariant. However, there exist an infinite sequence of functionals in $\mathcal{N}$ which tend to the exponential of the Gauss invariant.

The following is a possible generalization of the exponential of the Gauss invariant in the extended loop representation:

$$
\exp ^{*}(\boldsymbol{E}) \equiv \lim _{M \rightarrow \infty} F_{M}(\boldsymbol{E})
$$


where

$$
F_{M}(\boldsymbol{E}) \equiv \sum_{n=0}^{M} \frac{a^{n}}{2^{n} n !} g_{\mu_{1} \nu_{1}} \cdots g_{\mu_{n} \nu_{n}} E^{\left(\mu_{1} \nu_{1} \cdots \mu_{n} \nu_{n}\right)_{S}}
$$

and $g_{a x b y} \equiv-\varepsilon_{a b c} \partial_{c} \nabla^{-2} \delta(x-y)$ is the propagator of the Chern-Simons theory. In (31), the subscript ' $S$ ' indicates a sum over all the permutations of the Greek indexes. The functions $F_{M}(\boldsymbol{E})$ belong to $\mathcal{N}$, satisfy the Mandelstam identities (27) and, it can be shown that, they are diffeomorphism invariant. By evaluating (30) in loop coordinates, we get

$$
\exp ^{*}[\boldsymbol{X}(\Gamma)]=\exp [a \rho(\Gamma)]
$$

where $\rho(\Gamma)$ is the Gauss self-linking number of the loop $\Gamma$. In order to obtain (32), the following algebraic constraint, satisfied by the loop coordinate, was used,

$$
[\boldsymbol{X}(\Gamma)]^{\dot{\beta}_{1} \nu_{1} \cdots \dot{\beta}_{n} \nu_{n} \dot{\beta}_{n+1}} \delta_{\dot{\beta}_{1} \cdots \dot{\beta}_{n+1}}^{\mu_{1} \cdots \mu_{m}}=[\boldsymbol{X}(\Gamma)]^{\mu_{1} \cdots \mu_{m}}[\boldsymbol{X}(\Gamma)]^{\nu_{1} \cdots \nu_{n}}
$$

As shown in reference [10], $\exp [-3 \Lambda \rho(\Gamma) / 2]$ is a formal solution of gravity with cosmological constant in the loop representation. It is expected that its extension (30) is the corresponding solution in the extended loop representation. However, exp* does not converge for every vector $\boldsymbol{E}$. A possible solution to this problem would be to limit the extended loop space to those vectors that satisfy an algebraic constraint equal to (33). In this case, (30) converges to

$$
\exp ^{*}[\boldsymbol{E}]=\exp \left[\frac{a}{2} g_{\mu_{1} \mu_{2}} E^{\mu_{1}} E^{\mu_{2}}\right]
$$

The problem of the appropriate definition of $\exp ^{*}$ and the action of gravity constraints on this functional is still under study. It is interesting to point out that there are also problems in the lattice, where it has not been possible to define an exponential of the Gauss number which simultaneously satisfies the Hamilton and diffeomorphism constraint [11.

We wish especially to thank Rodolfo Gambini for his critical comments.

\footnotetext{
${ }^{1}$ For each $n, E^{\nu_{1} \cdots \nu_{n}}$ is a vector density of weight one in each argument $\nu=(a x)$.
} 


\section{References}

[1] A. Ashtekar, Phys. Rev. Lett. 57, 2244 (1986).

[2] A. Ashtekar, Phys. Rev. D36, 1587 (1987).

[3] C. Rovelli, L. Smolin, Phys. Rev. Lett. 61, 1155 (1988).

[4] C. Di Bartolo, R. Gambini and J. Griego. Comm. Math. Phys. 158, 217-240 (1993).

[5] C. Di Bartolo, R. Gambini, J. Griego and J. Pullin. Phys. Rev. Lett. 72, 3638 (1994).

[6] C. Di Bartolo, R. Gambini and J. Griego. Phys. Rev. D51, 502 (1995).

[7] C. Di Bartolo, R. Gambini, J. Griego and J. Pullin, J. Math. Phys. 36, 6510 (1995).

[8] T. Schilling, "Noncovariance of the extended holonomy: examples" preprint CGPG$95-/ 3-1$

[9] C. Di Bartolo and J. Griego, Phys. Lett. B317, 540 (1993).

[10] R. Gambini, J. Pullin, in "Knots and quantum gravity", J. Baez editor, Oxford University Press (1993).

[11] R. Gambini, private communication. 